“(C) 2015 IEEE. Personal use of this material is permitted. Permission from IEEE must be obtained for all other uses, in any current or future media, including reprinting/republishing this material for advertising or promotional purposes, creating new collective works, for resale or redistribution to servers or lists, or reuse of any copyrighted component of this work in other works." 


\section{A Passive Estimator of Functional Degradation in Power Mobility Device Users}

\author{
James Poon \\ Faculty of Engineering and IT \\ University of Technology, Sydney \\ james.poon@student.uts.edu.au
}

\author{
Jaime Valls Miro \\ Faculty of Engineering and IT \\ University of Technology, Sydney \\ jaime.vallsmiro@uts.edu.au
}

\author{
Ross Black \\ Occupational Therapy Department \\ Prince of Wales Hospital, Sydney \\ ross.black@sesiahs.health.nsw.gov.au
}

\begin{abstract}
This paper documents the development of a passive technique for assessing a power mobility device user's driving proficiency during everyday driving activities outside formal assessment conditions by therapists. This is approached by first building a model by means of an Artificial Neural Network to infer longer-term destinations for discretized bouts of travel, and subsequently drawing cues indicative of decline in driving proficiency for the duration of point-to-point navigation rather than relying on instantaneously calculated metrics. This resultant quantity, which we refer to as 'functional degradation', can then provide therapists with additional information concerning user health or serve as a leveraging parameter in combinatory sharedcontrol mobility frameworks. Experiments conducted by able-bodied users subject to simulated noise scaled to varying degrees of functional degradation reveal a quantitative correlation between these longer-term proficiency metrics and the magnitude of degradation experienced; a promising outcome that sets the scene for a larger-scale clinical trial.
\end{abstract}

\section{Motivation}

The use of power mobility devices (PMD's) becomes quite prevalent in developed countries for people over the age of 65 , with nearly 5 million in the United States alone [1]. Along with predictions that the global population of people aged over 60 is set to approximately double by 2050 [2] there is a drive to improve the means by which services in the field of aged and disabled care are administered, especially towards those still leading active lives in their communities [3]. This has manifested in a myriad of approaches including automated smart-homes [4], [5] and adaptations to facilities to better accommodate both disabled individuals [6] and their respective apparatus [7].

Yet matching the technological advances that make up modern PMDs to users needs is not a straightforward process. These are heavy devices capable of manoeuvring at fast speeds and operated both in- and out-doors, in the presence of static (architectural barriers) and dynamic (pedestrian and vehicular traffic) hazards. Common practice in power mobility aid assignment is manual examination through a series of scripted navigation tasks [8] at the therapist's facilities. The ability of observing the client using the device in the environments in which it is to be used during a limited time is also often undertaken by a therapist during a typical PMD assessment. However that represents a snapshot of the user abilities under fairly prescribed conditions. On-going monitoring of the users' proficiency is time-consuming for both users and therapists, and hardly possible in practical terms. Moreover, the amount of data gather would generally be very large and impose severe constraints on how a therapist could analyse it efficiently. In this work, a mechanism to extract a measure of functional degradation from longterm monitoring is proposed, i.e. a quantitative indicator of decline in motor control in elderly and disabled individuals. This is viewed as a useful mechanism for therapists and carers to gain an insight into supplementary data from longer-term health monitoring when steadily available for the purpose of identifying peaks and trends, as well as additional evidence-based information that can be taken into consideration by a therapist when re-considering the prescription of the use of specific PMDs on an on-going basis.

\section{INTRODUCTION AND BACKGROUND}

There have been multiple approaches towards autonomous assessment of PMD users given intelligent mobility aids [9]. Our earlier work [10] aimed to closely replicate manual assessment through standard tasks such as driving 10 metres forward, and grading taking place by means of various machine learning algorithms. Profiling capabilities of wheelchair users in an assessment-style environment have also been proposed by Urdiales et al. [11], providing therapists with information concerning particular driving maneuvre deficiencies. These attributes can be used to aid in determining whether or not an individual is suited to a PMD, however as interest in intelligent PMD systems is increasing [12], measures of proficiency have also been used in providing a user share in combinatory human-machine control protocols for increased safety and travelling comfort [13]-[15]. An important benefit of utilising autonomous means of inferring proficiency metrics to complement therapists's own assessment is the mitigation of human inconsistencies that may accompany manual assessment by therapists [16], as well as the ability to review and revisit the assessment data at their own leisure.

Passively measuring this degradation factor through everyday practices that the user would perform regardless of being under assessment provides a more candid and 


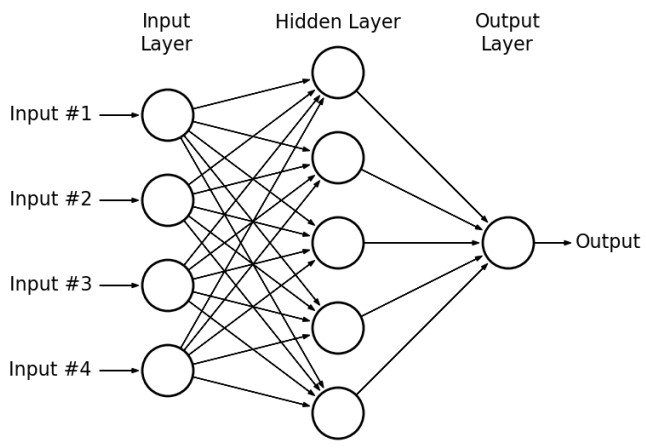

Fig. 1. Example of a feed-forward Artificial Neural Network [22]

realistic measurement of user health, compared to assessment conditions which may feel stressful and unfamiliar. Longer-term monitoring has been approached by means of installing unobtrusive sensors in homes [17], regular surveys [18] or telemonitoring via regular cellular text messages [19], [20]. By contrast, little work has been done in constant background assessment of user ability and declines or improvements in their level of health within a mobility-specific context. To this end, a methodology is proposed to draw correlations between a PMD user's level of functional degradation during bouts between discrete locations, with metrics that are measured over the course of each bout instead of instantaneously acquired values. The primary advantage of this approach is its ability to be carried out in settings familiar to the user, and towards obtaining steady, longer-term quantifiable measurements of PMD proficiency as an indicator of overall health through everyday activity as opposed to 'snapshots' of user ability during assessment time which may not be indicative of true proficiencies.

The remainder of this paper is as follows: a description of the process suggested to assess functional degradation is explained in Section III, with the experimental setup on an electric wheelchair given in Section IV. Preliminary results are covered in Section $\mathrm{V}$ to show the validadity of the approach in an indoor setting, with concluding remarks compiled in Section VI.

\section{PROPOSITION}

This work proposes a two-step mechanism to assess long-term degradation:

- a statistical learning tool to identify implicit bout destinations by the user of the PMD

- a metric for functional degradation derived over the inferred mobility bouts

\section{A. Artificial Neural Networks}

In order to obtain a measurement of user proficiency without explicit prior information about their longer-term destination, an Artificial Neural Network (ANN) [21] is proposed. ANNs are cognitive algorithms that attempt

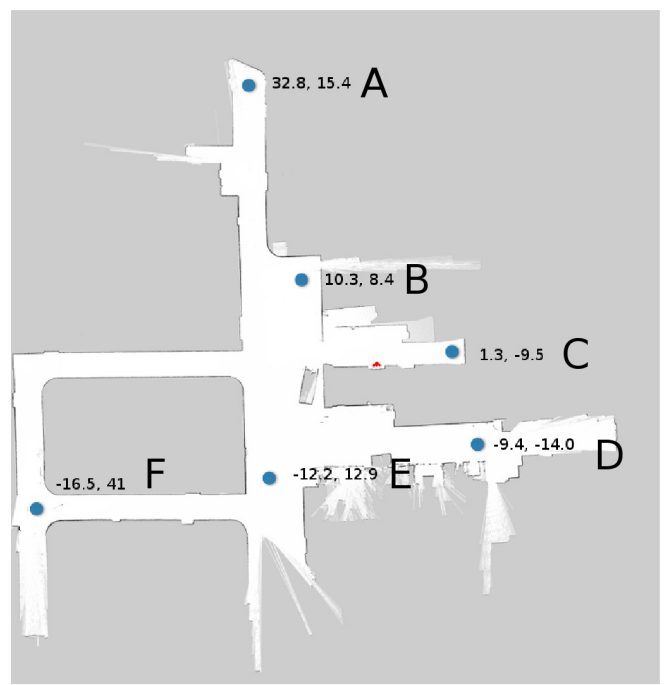

Fig. 2. Test environment with origin in red, and destinations in blue (coordinates in meters, approx size $40 \mathrm{~m}^{2}$ )

to replicate brain neuron behaviour in order to provide a machine learning mechanism for universal function approximation, which can take the form of regression or classification problems. A key factor of interest in these models is their ability to "learn" from input data, so that they can be re-trained in an efficient manner as more data becomes available. Figure 1 shows an example of a small ANN.

For the work hereby proposed, training data consisted of six runs to each one of six possible destination of interest arbitrarily chosen in an indoor environment, hence a total of thirty six healthy training examples (the experimental set-up used in this work is described in more detail in Section IV). A 2D representation of the environment used in the PMD navigational exercises is depicted in Figure 2. The output of this ANN takes the form of six likelihoods $[0,1]$, giving the ANN the ability to provide an indication of certainty about the destination likelihoods (rather than discrete discriminative outputs). Figure 3 shows an example of destination convergence over the duration of a single bout for an environment with the six possible destinations. In this example it can be see how the uppermost destination $\mathrm{A}$ is the more likely destination chosen by the user.

The input features to the network were chosen in order to best provide the ANN with information related to both the platform's position and movement in the known envrionment, as well as the continuous input behaviour from the user as exercised through the driving joystick controller. This is an important aspect of this work as it allows the network to provide an instantaneous likelihood distribution across the allocated destination points, for actionable destination data beginning with early-stage movement from rest. The ANN was thus trained using the following features to determine destination likelihoods:

- Joystick displacement: $\left(j o y_{x}, j o y_{y}\right)$ representing 


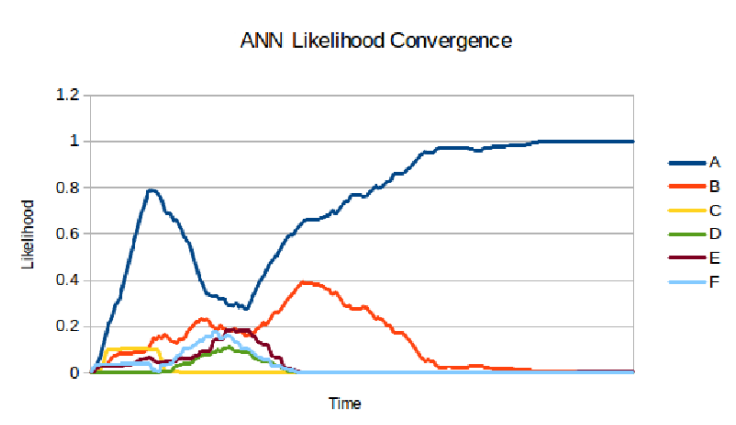

Fig. 3. Sample destination likelihood convergence. Destinations A-F are in clockwise order from the uppermost destination in Fig 2

TABLE I. MEAN Bout Durations

\begin{tabular}{c|c}
\hline Destination & Mean Bout Duration (s) \\
\hline A & 52 \\
B & 33 \\
C & 16 \\
D & 52 \\
E & 31 \\
F & 63 \\
\hline
\end{tabular}

forward/backward control inputs, and left/right commands respectively

- Global wheelchair position in the mapped environement $(x, y, \theta)$

- Instantaneous wheelchair linear and angular velocities $(v, w)$

\section{B. Functional Degradation}

In this work we define functional degradation as a percentage. $0 \%$ denotes a healthy user baseline, yet it could have equally been set to any other instant in time from which to measure degradation. Higher percentages thus indicate more severe deficiencies. In order to quantify this degradation we use two metrics measured over the entirety of each bout: jerkiness, and the time taken in comparison to that of a healthy (baseline) user. In these experiments, the datum time was taken to be the mean of the ANN training data durations for each destination (Table I).

Jerkiness thus represents a scaled measure of the magnitude of the wheelchair's rotational speed changes averaged over bout duration, as given by

$$
\text { Jerkiness }=C_{j} \times \frac{\Sigma|\Delta \omega|}{\text { duration }_{\text {bout }}}
$$

The coefficient $C_{j}$ is simply a constant factor to bring the two metrics into scale.

\section{EXPERIMENT SETUP AND RESULTS}

Testing the relationship between our two metrics and functional degradation was carried out on an instrumented wheelchair, depicted in Figure 4, fitted with wheel encoders and serial control interface. An additional sensor module was added, containing a MS Kinect RGBD camera, Hokuyo planar laser scanner and an Xsens

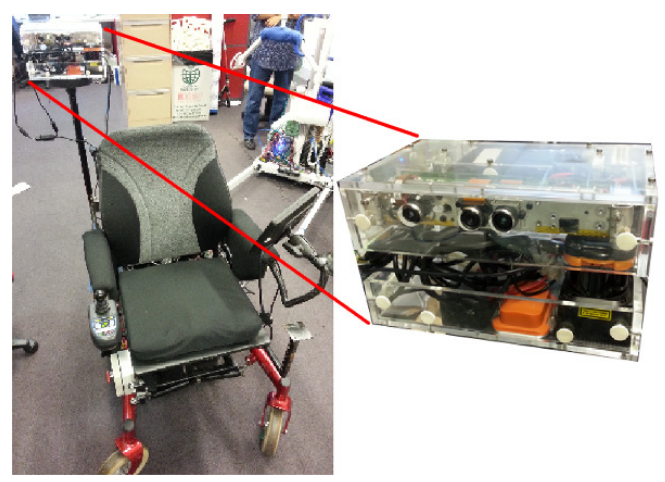

Fig. 4. Instrumented wheelchair with mounted sensor package

inertial measurement unit. More information covering the details of the sensor module can be found in [23]. For the test environment, a basement level of UTS was used (Fig 2). This map was generated using Hector SLAM [24] (Simultaneous Localization and Mapping) on laser scanner data from the module. Each laser scan consists of several hundred range readings across a 180 degree sweep of the sensor's horizontal field of view. Experimentation was instrumented via the Robot Operating System (www.ros.org). The red triangle indicates the map origin, with six designated points of interest marked with blue circles. White space represents the traversable floorspace, light grey areas represent inaccessible areas and the black borders indicate obstacles such as walls and other artefacts. The entirety of the traversable test area measures approximately 40 metres square.

Taking the trained ANN, five able-bodied users were instructed to drive to each destination while having their joystick inputs subject to random zero-mean Gaussian noise scaled to varying magnitudes of functional degradation. The $\sigma$ of this noise was set prior to each trial by $\sigma=F . D . \times \sigma_{\max } . \sigma_{\max }$ was empirically set to 0.5 ; for example at F.D. $=50 \%, \sigma=0.25$. This noise was then merged with the user joystick input as per Algorithm 1. No noise is added if the joystick is at rest, in accordance to zero movement when the user releases the joystick.

$$
\begin{aligned}
& \text { if } j o y_{x} \neq 0 \| j o y_{y} \neq 0 \text { then } \\
& \begin{array}{l}
j o y_{x}=j o y_{x}+N_{\text {rand }}(0, \sigma) \\
j o y_{y}=j o y_{y}+N_{\text {rand }}(0, \sigma) \\
\text { limit } j o y_{x}, j o y_{y} \text { to } \pm 1.0
\end{array} \\
& \text { end }
\end{aligned}
$$

Algorithm 1: Gaussian noise addition

The scaling coefficient $C_{j}$ was set to 100 for visualisation purposes. For destinations $\mathrm{A}$ to $\mathrm{D}$, five bouts were recorded for each tier of increasing simulated functional degradation from $0 \%$ to $50 \%$, with a step of $10 \%$. Figures 7-10 show averaged plots of jerkiness and bout duration over gradual increases in functional degradation, demonstrating a quantitative correlation between functional degradation and the two aforementioned metrics. Figures 5 and 6 demonstrate the effects of Gaussian noise 


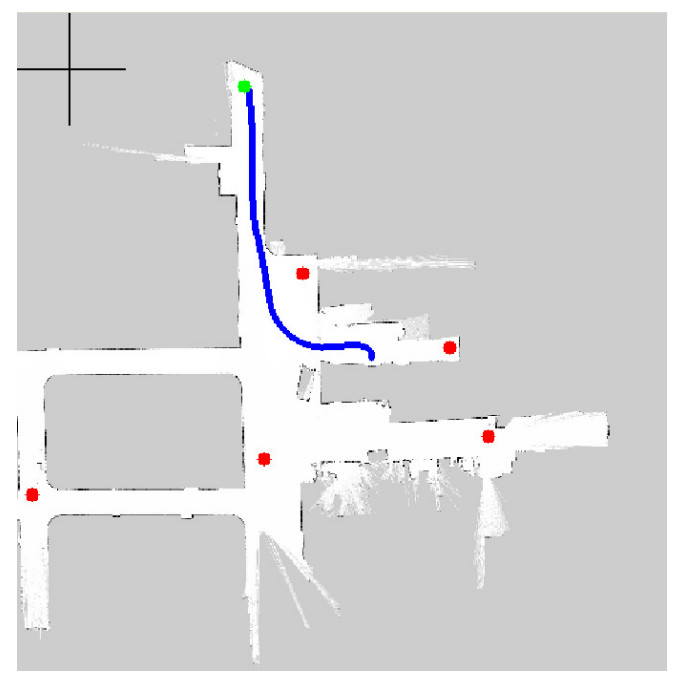

Platform Velocity Profile

$0 \%$ functional degradation

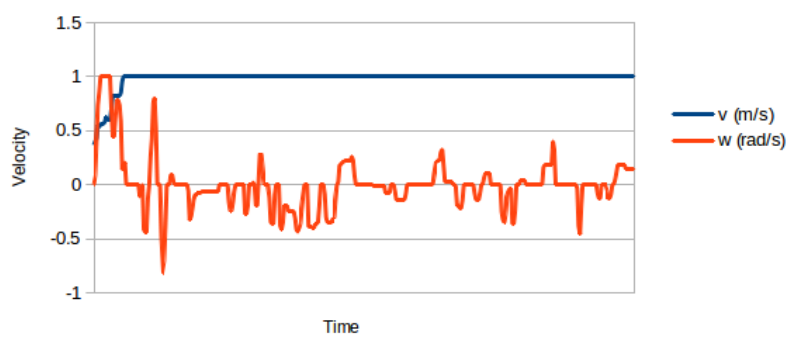

Fig. 5. Able-bodied (F.D. $=0 \%$ ) trajectory and velocity profile to destination $\mathrm{A}$

addition on driving behaviour to destination A.

\section{DisCUSSION AND FUtURE WORK}

At functional degradation values beyond $50 \%$, manoeuvring the wheelchair became impractical, and likely beyond the comfort zone for a frail user especially over longer-term usage periods. Tables II-V, averaged in graphical form in Figure 11 shows the gradual increase in convergence time as functional degradation increases; towards $50 \%$ F.D., convergence does not occur until a significant proportion of the bout is already completed, making the ANN less useful as an inference mechanism without prior destination data under severe degradation. However, at this stage it is unlikely an individual would use PMDs under their own control and would almost certainly require alternate mobility means. Adaptation of features from our earlier user grading experiments [10] may also provide additional insights concerning user health, as well as additional parameters to therapists in order to augment conventional pen-and-paper assessment sessions.

Future work will first focus on developing the ANN to recognise bouts between destination points, rather than only from the origin designated in the test area. The jerk metric may be more informative considering deviations in both linear and angular joystick inputs. Due to individual

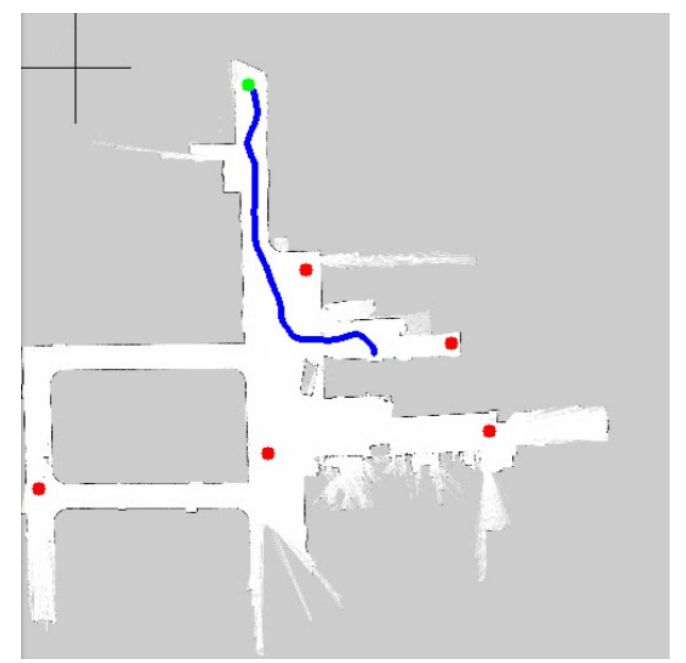

Platform Velocity Profile

$50 \%$ functional degradation

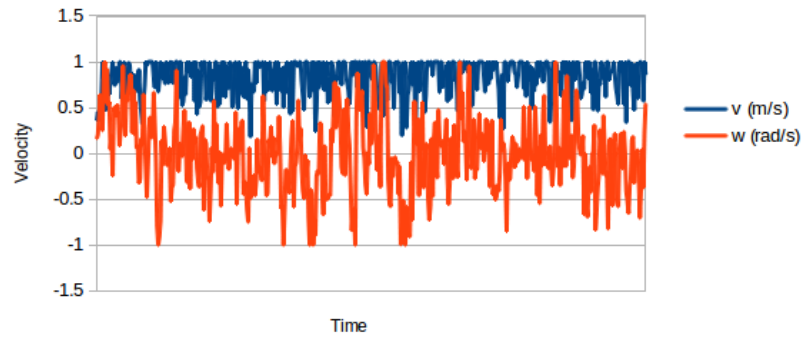

Fig. 6. Trajectory and velocity profile under simulated disability $(F . D .=50 \%)$ to destination A

disabilities affecting specific muscle groups, future simulacra of disabilities should also take into account nonzero mean additive noise models. The possibility that a user may wish to pause on the way or take a deviant route, or bouts to yet-unknown destinations will need to be integrated into further developments. Additional contextual likelihood priors such as the time of day [25] can also be implemented in order to bolster the inference mechanism, which can be paired with shared control strategies and the like.

Despite the shortcomings that arise from limited trials across a relatively small group of able-bodied test users, this outcome appears promising in advancing the longer-term monitoring of PMD user health and driving proficiency by stepping away from conventional assessment techniques such as surveys and physical visits to/by therapists. Access to constantly updating data concerning patient welfare could serve as a supplement to scheduling therapist intervention according to the health of the patient, rather than to a regulated schedule. This has the benefit of saving time and cost, as physical therapist visits are time intensive, costly and may be strenuous to the elderly or disabled PMD user.

Acute teaching hospitals in Australia and likely in many other countries dedicate substantial time and re- 
TABle II. 90\% Likelihood Convergence Time (s) - Dest. A

\begin{tabular}{c||c|c|c|c|c}
\hline \multicolumn{1}{l||}{ F.D. (\%) } & 1 & 2 & 3 & 4 & 5 \\
\hline 0 & 21.0 & 19.2 & 18.5 & 22.3 & 23.7 \\
10 & 20.2 & 20.3 & 19.6 & 21.8 & 17.5 \\
20 & 23.0 & 22.3 & 24.4 & 21.5 & 23.0 \\
30 & 24.3 & 23.8 & 25.1 & 22.4 & 26.0 \\
40 & 24.7 & 24.3 & 25.3 & 23.9 & 26.2 \\
50 & 26.2 & 25.6 & 26.8 & 25.1 & 27.7 \\
\hline
\end{tabular}

TABLE III. 90\% LiKelihood CONVERgence Time (S) - Dest.

\begin{tabular}{c||c|c|c|c|c}
\hline \multicolumn{1}{c||}{ F.D. (\%) } & \multicolumn{5}{c}{ Trial } \\
& 1 & 2 & 3 & 4 & 5 \\
\hline 0 & 12.3 & 12.0 & 11.2 & 14.1 & 13.0 \\
10 & 14.2 & 12.9 & 14.3 & 15.1 & 13.6 \\
20 & 14.9 & 16.6 & 13.2 & 14.7 & 15.2 \\
30 & 16.1 & 16.3 & 18.5 & 16.0 & 14.8 \\
40 & 16.5 & 19.4 & 16.3 & 16.9 & 13.4 \\
50 & 16.8 & 15.0 & 17.0 & 18.1 & 15.3 \\
\hline
\end{tabular}

Fig. 7. Jerkiness and bout duration vs functional degradation - Dest. A

Functional Degradation vs Jerkiness and Bout Duration

Destination B

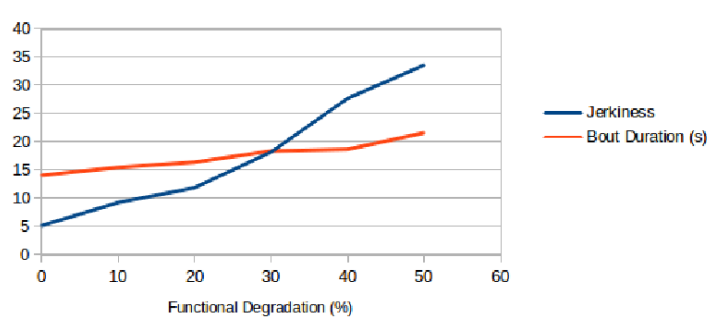

Fig. 8. Jerkiness and bout duration vs functional degradation - Dest. B

Functional Degradation vs Jerkiness and Bout Duration

Destination $\mathrm{C}$

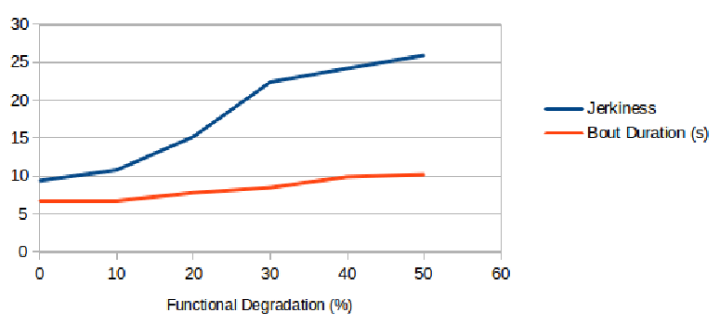

Fig. 9. Jerkiness and bout duration vs functional degradation - Dest. C

Functional Degradation vs Jerkiness and Bout Duration

Destination $D$

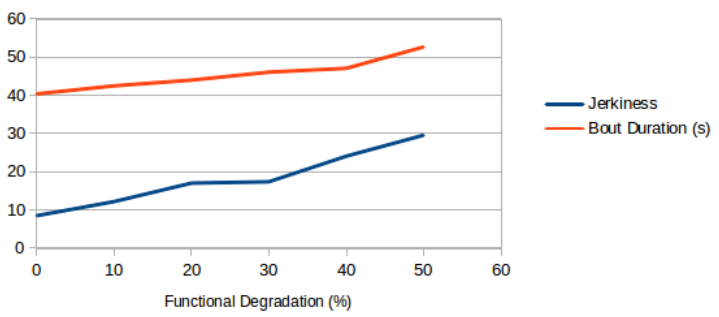

Fig. 10. Jerkiness and bout duration vs functional degradation - Dest.

$\mathrm{C}$

\begin{tabular}{c||c|c|c|c|c}
\hline \multicolumn{1}{c||}{ F.D. (\%) } & \multicolumn{5}{c}{ Trial } \\
& 1 & 2 & 3 & 4 & 5 \\
\hline 0 & 3.3 & 2.9 & 4.3 & 3.8 & 2.3 \\
10 & 3.2 & 4.7 & 2.8 & 2.6 & 2.1 \\
20 & 3.8 & 4.1 & 5.3 & 2.4 & 2.7 \\
30 & 3.6 & 2.4 & 4.8 & 4.8 & 4.1 \\
40 & 3.7 & 6.3 & 1.7 & 4.8 & 2.9 \\
50 & 4.6 & 4.3 & 4.4 & 5.8 & 3.2 \\
\hline
\end{tabular}

TABle V. 90\% Likelihood Convergence Time (s) - Dest. D

\begin{tabular}{c||c|c|c|c|c}
\hline \multicolumn{1}{l||}{ F.D. (\%) } & \multicolumn{5}{c}{ Trial } \\
& 1 & 2 & 3 & 4 & 5 \\
\hline 0 & 20.1 & 17.6 & 19.6 & 22.1 & 20.2 \\
10 & 21.6 & 21.0 & 23.2 & 20.4 & 21.5 \\
20 & 21.8 & 20.0 & 25.0 & 22.4 & 22.1 \\
30 & 25.9 & 23.6 & 26.5 & 23.6 & 28.5 \\
40 & 25.7 & 27.7 & 26.8 & 23.4 & 26.1 \\
50 & 28.0 & 26.3 & 29.8 & 26.3 & 27.7 \\
\hline
\end{tabular}

sources to programs that aim to reduce admission rates and length of stay (LOS) as an acute hospital bed is a limited and expensive resource. If a reduction in a patient's status could be identified by changes in their functional performance as determined by an intelligent monitoring system, then a visit by qualified health or medical/nursing staff could be sufficient to ameliorate their condition before they reach a crisis and require admission. Alternatively, the patient could be identified earlier in their decline, and if a hospital stay was needed it would potentially be shorter than if the patient was present with an advanced condition. As a result the patient is also saved the disruption and anxiety associated with a hospital admission.

\section{CONCLUSION}

The paper describes an approach to interpret pointto-point driving data from a standalone sensor module mounted on an electric wheelchair (or other PMD) in order to assess a quantity of decline in independent mobility proficiency, which we have termed as functional degradation. This technique is of potential use to therapists and other staff involved in the everyday care and monitoring of disabled and elderly individuals reliant on PMDs for routine locomotion; data gleaned from movement bouts in 
Destination Convergence Time vs Functional Degradation

go\% likelihood threshold

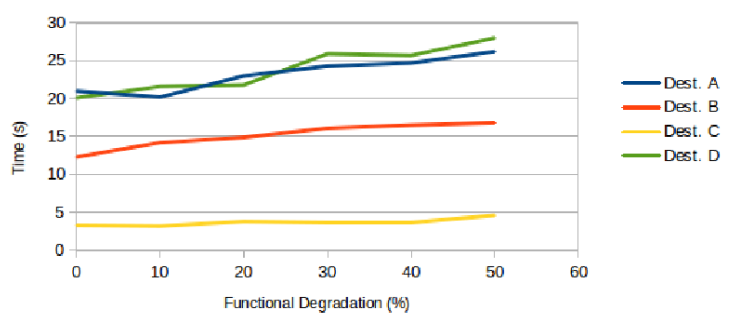

Fig. 11. Averaged $90 \%$ likelihood convergence times

the users' own place of residence can be critically analyzed to provide insights into the functional degradation status without the need for a physical meeting to occur. Our preliminary experiments indicate a quantitative correlation between our two metrics of input jerk and the time required to complete a discrete bout of travel, and the level of functional degradation expressed by the user. This outcome thus presents therapists with additional utilities to help discern whether or not user health is in decline, and if they can be continued to be permitted to have independent access to PMDs for mobility purposes within their community without presenting a risk to themselves or others. Despite the shortcomings of the experiments conducted we believe the results remain relevant within the context of this research, and hope to continue with a broad test user base of disabled individuals under the oversight of trained therapist staff to further develop this technique towards a more thorough functional degradation monitoring system within an assisted mobility scope.

\section{REFERENCES}

[1] H. S. Kaye, T. Kang and M. P. LaPlante, Mobility device use in the united states. Disability statistics report 14. Disability Statistics Center, University of California, San Francisco. (2000)

[2] Congressional Budget Office (C. B. O.), Global population aging in the 21st century and its economic implications. (2005) http://www.cbo.gov/sites/default/files/cbofiles/ ftpdocs/69xx/doc6952/12-12-global.pdf. Accessed: Mar 23, 2015.

[3] P. Clarke and A. Colantonio, Wheelchair use among community dwelling older adults: prevalence and risk factors in a national sample Canadian Journal on Aging, vol. 24, pp. 191198, 2005.

[4] J. B. Cheatham, M. A. Regalbuto, T. A. Krouskop and D. J. Winningham, A robotic system for improved living by severely disabled persons Intelligent Robots, 1988., IEEE International Workshop on, vol., no., pp.79,82, 1988.

[5] V. Santos, P. Bartolomeu, J. Fonseca, and A. Mota, B-live - a home automation system for disabled and elderly people Industrial Embedded Systems, 2007. SIES '07. International Symposium on, pp.333,336, 2007.

[6] N. A. Karim and C. Nwagboso, Assistive technologies in public transport: meeting the needs of elderly and disabled passengers Information and Communication Technologies: From Theory to Applications, 2004. Proceedings. pp.69, 2004.

[7] C. Harrison, M. Grant and B. Conway, Haptic interfaces for wheelchair navigation in the built environment Presence, vol.13, no.5, pp.520,534, 2004 .
[8] R. L. Kirby, J. Swuste, D. J. Dupuis, D. A. MacLeod, and R. Monroe, The wheelchair skills test: A pilot study of a new outcome measure. Archives of Physical Med. and Rehab., vol. 83, no. 1, pp. 1018, 2002.

[9] J. V. Miro, R. Black, B. Andonovski and G. Dissanayake, Development of a novel evidence-based automated powered mobility device competency assessment. IEEE 13th International Conference on Rehabilitation Robotics (ICORR), pp1-8, 2013.

[10] B. Andonovski, J. V. Miro, J. Poon and R. Black, An automated mechanism to characterize wheelchair user performance. Biomedical Robotics and Biomechatronics (2014 5th IEEE RAS \& EMBS International Conference on), pp.444-449, 2014.

[11] C. Urdiales, E. Perez, G. Peinado, M. Fdez-Carmona, J. Peula, R. Annicchiarico, F. Sandoval, and C. Caltagirone, On the construction of a skill-based wheelchair navigation profile. IEEE Trans. on Neural Systems and Rehab. Eng., vol. 21, no. 6, pp. 917-927, Nov 2013.

[12] P. Viswanathan, J. J. Little, A. K. Mackworth, and A. Mihailidis, Navigation and obstacle avoidance help (noah) for older adults with cognitive impairment: A pilot study ACM SIGACCESS Conf. on Computers and Accessibility, 2011, pp. 4350.

[13] Q. Li, W. Chen and J. Wang, Dynamic shared control for humanwheelchair cooperation. Robotics and Automation (ICRA), 2011 IEEE International Conference on. pp. 4278-4283, 2011.

[14] C. Urdiales, J. M. Peula, M. Fdez-Carmona, C. Barru, E. J. Prez, I. Snchez-Tato, J. C. Toro, F. Galluppi, U. Corts, R. Annichiaricco, C. Caltagirone and F. Sandoval, A new multi-criteria optimization strategy for shared control in wheelchair assisted navigation. Autonomous Robots. vol. 30, no. 2, pp. 179197, 2011.

[15] J. Poon and J. V. Miro, A multi-modal utility to assist powered mobility device navigation tasks. Social Robotics. vol. 8755, pp. 300-309, Springer International Publishing, 2014.

[16] W. B. Mortenson, W. C. Miller and C. Auger, Issues for the selection of wheelchair-specific activity and participation outcome measures: a review. Archives of Physical Med. and Rehab., vol. 89, no. 6, pp. 1177-1186, 2008.

[17] M. Ogawa, R. Suzuki, S. Otake, T. Izutsu, T. Iwaya and T. Togawa, Long term remote behavioral monitoring of elderly by using sensors installed in ordinary houses. Microtechnologies in Medicine \& Biology 2nd Annual International IEEE-EMB Special Topic Conference on , vol., no., pp.322,325, 2002.

[18] M. Lagergren, ASIM: a system for monitoring and evaluating the long-term care of the elderly and disabled. Health Services Research. 28(1):27-44, 1993.

[19] C. N. Scanaill, B. Ahearne and G. M. Lyons, Long-term telemonitoring of mobility trends of elderly people using SMS messaging. IEEE Trans Inf Technol Biomed. 10(2):412-3, 2006.

[20] D. Barrett, The role of telemonitoring in caring for older people with long-term conditions. Nurs Older People. 24(7):21-5, 2012.

[21] S. B. Maind and P. Wankar, Research paper on basic of artificial neural network. International Journal on Recent and Innovation Trends in Computing and Communication, vol. 2, no. 1, pp. 96100, 2014.

[22] J. VanderPlas, Neural network diagram (2013) http: / /www.astroml.org/book_figures/appendix/ fig_neural_network.html. Accessed: Mar 24, 2015.

[23] J. V. Miro, R. Black, F. de Bruijn, and G. Dissanayake, Semiautonomous competency assessment of powered mobility device users. IEEE Intl. Conf. on Rehab. Robotics, 2011, pp. 16.

[24] S. Kohlbrecher, J. Meyer, O. von Stryk, and U. Klingauf, A flexible and scalable slam system with full $3 d$ motion estimation. IEEE Intl. Symp. on Safety, Security, and Rescue Robotics, pp. 155160, 2011.

[25] C. Phua et al., 2-layer erroneous-plan recognition for dementia patients in smart homes. e-Health Networking, Applications and Services, 2009. Healthcom 2009. 11th International Conference on , pp.21,28, 2009. 